B. Dorfmeister $\cdot$ S. Brandlhofer · F. G. Schaap •

M. Hermann · C. Fürnsinn · B. P. Hagerty

H. Stangl $\cdot$ W. Patsch $\cdot$ W. Strobl

\title{
Apolipoprotein AV does not contribute to hypertriglyceridaemia or triglyceride lowering by dietary fish oil and rosiglitazone in obese Zucker rats
}

Received: 24 June 2005 / Accepted: 14 December 2005 / Published online: 29 March 2006

(C) Springer-Verlag 2006

\begin{abstract}
Aims/hypothesis: Apolipoprotein AV (apoAV) is a recently discovered apolipoprotein with a triglyceridelowering effect in genetically modified mice. Transcription of the human gene encoding apoAV (APOA5) is suppressed by insulin and stimulated by fibrates. Our goal was to study the expression of Apoa5, in comparison with Apoa4 and Apoc3, in hypertriglyceridaemic, obese and insulin-resistant Zucker rats receiving the insulin sensitiser rosiglitazone and/or a fish oil diet to lower triglycerides. Methods: Hepatic Apoa5, Apoa4 and Apo3 mRNA and liver and plasma apoAV were measured in lean and obese Zucker rats receiving rosiglitazone while on a coconut oil or fish oil diet. Results: Basal hepatic Apoa5 expression was similar in obese and lean Zucker rats. Unexpectedly, obese Zucker rats tended to have higher plasma apoAV levels despite their hypertriglycer-
\end{abstract}

B. Dorfmeister · S. Brandlhofer · B. P. Hagerty ·

H. Stangl - W. Strobl $(\bowtie)$

Department of Medical Chemistry,

Center of Physiology and Pathophysiology,

Medical University of Vienna,

Währinger Strasse 9,

A-1090 Vienna, Austria

e-mail: wolfgang.strobl@meduniwien.ac.at

Tel.: +43-4277-60822

Fax: +43-4277-60881

M. Hermann

Department of Medical Biochemistry,

Medical University of Vienna,

Vienna, Austria

C. Fürnsinn

Department of Internal Medicine III,

Medical University of Vienna,

Vienna, Austria

W. Patsch

Institute for Laboratory Medicine,

Paracelsus Private Medical University,

Salzburg, Austria

F. G. Schaap

AMC Liver Centre,

Amsterdam, The Netherlands idaemic state. Both rosiglitazone and the fish oil diet significantly increased Apoa 5 mRNA, by about $70 \%$, but tended to lower liver and plasma apoAV. Rosiglitazone had no effect on Apoa 5 mRNA in cultured rat hepatocytes. No intact PPAR (peroxisome proliferator-activated receptor) response element was identified in the rat Apoa5 promoter. Conclusions/interpretation: Our data indicate that apoAV does not contribute to the hypertriglyceridaemia of obese Zucker rats or to the hypolipidaemic effect of rosiglitazone or a fish oil diet. The divergent changes of Apoa5 mRNA and apoAV levels suggest co- or posttranslational regulation. The increase in Apoa 5 mRNA induced by rosiglitazone is not directly mediated by peroxisome proliferator-activated receptor $\gamma$.

Keywords Apolipoprotein AV · Apolipoprotein A-IV • Apolipoprotein C-III - Rosiglitazone - Fish oil diet . Zucker rats $\cdot$ Hypertriglyceridaemia

Abbreviations apo: apolipoprotein PPAR: peroxisome proliferator-activated receptor - PPRE: PPAR response element $\cdot$ PUFA: polyunsaturated fatty acids

\section{Introduction}

Apolipoprotein AV (apoAV), a recently discovered member of the apolipoprotein family, is considered to play an important role in plasma triglyceride transport $[1,2]$. The gene for apoAV (APOA5) is part of the APOA1/A4/C3/A5 gene cluster on chromosome 11 in man, and $A P O A 5$ is predominantly expressed in the liver. Human APOA5 polymorphisms are associated with elevated plasma triglyceride levels [2-4]. Studies in genetically modified mice and adenovirus overexpression experiments revealed a strong triglyceride-lowering effect of apoAV [2, 5-7]. This effect involves the stimulation of lipoprotein lipase activity and possibly inhibition of hepatic VLDL-triglyceride secretion [5-8]. ApoAV is considered to have effects opposite to those of apoC-III in triglyceride metabolism, as shown by studies in genetically modified mice (reviewed in 
[9]). Agonists of peroxisome proliferator-activated receptor $\alpha(\operatorname{PPAR} \alpha)$ stimulate the transcription of human APOA5 and increase serum apoAV levels in cynomolgus monkeys, suggesting a contribution of apoAV to the hypotriglyceridaemic effect of these drugs [10-12]. Human APOA5 transcription is repressed by liver $\mathrm{X}$ receptor agonists and insulin $[13,14]$. ApoA-IV, the apolipoprotein most closely related to apoAV $[1,2]$, participates in reverse cholesterol transport as an acceptor of cellular cholesterol and activator of LCAT (lecithin:cholesterol acyltransferase) $[15,16]$. ApoA-IV exerts a strong anti-atherogenic effect [17] and has also been suggested to act as a satiety factor. Plasma apoA-IV is elevated in human type 2 diabetes and obesity and decreases during weight reduction $[18,19]$.

falfa Zucker rats develop severe obesity due to the missense mutation fatty (Glu269Pro) in the extracellular domain of the leptin receptor. Obese Zucker rats are widely used as a model for the hypertriglyceridaemia and insulin resistance associated with human obesity [20]. The hypertriglyceridaemia of obese Zucker rats results from increased hepatic VLDL production [21]. Although subject to controversy $[6,8]$, this step in lipoprotein metabolism has been shown to be influenced by apoAV [5]. The severe insulin resistance in obese Zucker rats can be alleviated by rosiglitazone and other PPAR $\gamma$ agonists, which are used to treat human type 2 diabetes [22]. The resulting improvement in insulin sensitivity is associated with a marked decrease in plasma triglycerides. In addition to pharmacological interventions, the increased plasma triglycerides in obese Zucker rats can be reduced by fish oil diets rich in $\omega$ 3 polyunsaturated fatty acids (PUFA) [23-25]. These diets reduce hepatic lipogenesis and VLDL synthesis by incompletely understood mechanisms and increase fatty acid oxidation via activation of $\operatorname{PPAR} \alpha$ (reviewed in [26]). Fish oil diets markedly reduce the transcription of Apoal and Apoa4 in rat liver without affecting Apoc3 mRNA abundance $[24,25,27]$. It is unknown whether the expression of Apoa5 is altered by dietary $\omega-3$ PUFA and whether apoAV may contribute to their triglyceride-lowering effect.

In the liver of obese Zucker rats and other rat models of obesity, Apoa4 mRNA is increased [24, 25, 28, 29], whereas Apoc 3 mRNA levels are similar in lean and obese Zucker rats [25]. Dose-dependent inhibition of apoA-IV production in rat hepatocyte cultures by insulin has been described [30], suggesting that the overexpression of Apoa4 in the liver of obese rats may be related to insulin resistance. Rosiglitazone does not alter hepatic Apoa4 or Apoc3 expression in lean rats [31], but its effect on the increased Apoa4 mRNA in the liver of obese rats is not known.

The present study was designed to (1) determine the basal expression of Apoa5 in hypertriglyceridaemic, insulin-resistant obese Zucker rats; (2) determine whether Apoa 5 expression in these animals is altered when insulin resistance is improved and/or triglycerides are lowered by rosiglitazone or fish oil diet; and (3) study the effect of rosiglitazone on the increased expression of Apoa4 in the liver of obese Zucker rats.

\section{Materials and methods}

Experimental animals

Male $(F a /-)$ lean and ( $f a / f a)$ obese Zucker rats, $8-10$ weeks of age, were purchased from Harlan Winkelmann (Borchen, Germany) and housed in a room with a 12-h light 12-h dark cycle (lights on from 06.00 to $18.00 \mathrm{~h}$ ). Animal experiments, performed in accordance with the Principles of Laboratory Animal Care (NIH Publication No. 52-23 revised 1985) and with the Austrian Law on the Protection of Animals, were approved by the Animal Research Committee of the Medical University of Vienna.

Experimental diets and rosiglitazone treatment

The control diet (High Saturated Fat Diet; ICN, Cleveland, $\mathrm{OH}, \mathrm{USA}$ ) contained $20 \%(\mathrm{wt} / \mathrm{wt}$ ) coconut oil (main fatty acids, C12:0, lauric, 45.7\%; C14:0, myristic, $17.3 \%$; C16:0, palmitic, $8.8 \%$; C18:0, stearic, $12.0 \%$ ). The fish oil diet (High Menhaden Oil Diet; ICN) contained 20\% (wt/wt) Menhaden oil (main fatty acids, C16:0, palmitic, 15.2\%; C16:1, palmitoleic, 11.6\%; C18:1, oleic, 9.5\%; C18:2, linoleic: $1.8 \%, \mathrm{C} 18: 3$, linolenic, $1.8 \%$, C20:4, arachidonic, $2.3 \%, \mathrm{C} 20: 5$, eicosapentaenoic, $16.0 \%$, docosahexaenoic, $\mathrm{C} 22: 6,10.8 \%)$. The fish oil diet was stored in aliquots under nitrogen at $-80^{\circ} \mathrm{C}$ to avoid autooxidation. Rats received fresh aliquots daily. Rosiglitazone tablets (Avandia; GlaxoSmithKline, Uxbridge, UK) were pulverised and admixed to the diet at a concentration of $0.003 \% \mathrm{wt} / \mathrm{wt}$, resulting in a rosiglitazone intake in the range of 3-4 $\mathrm{mg} \mathrm{kg}^{-1}$ day $^{-1}$.

\section{Experimental design}

To study the effects of dietary $\omega-3$ PUFA, rosiglitazone and a combination of them, lean and obese Zucker rats were fed a control diet or fish oil diet ad libitum and/or received rosiglitazone as indicated in Table 1. Food was removed at $07.00 \mathrm{~h}$. Animals were killed $2-5 \mathrm{~h}$ later by sodium pentobarbital injection ( $5 \mathrm{mg} / 100 \mathrm{~g}$ i.p.), and liver and blood samples were collected.

\section{Tissue culture}

Primary rat hepatocyte cultures were prepared from adult male Sprague-Dawley rats using the collagenase perfusion technique [32]. Cells were plated at a density of $5 \times 10^{6}$ cells $/ 30 \mathrm{~mm}$ dish on a fibronectin matrix in DMEM, $4 \mathrm{mmol} /$ 1 glutamine, $10 \mathrm{mmol} / 1 \mathrm{HEPES}, \mathrm{pH} 7.6,10 \% \mathrm{FCS}$, and 
Table 1 Experimental design

\begin{tabular}{llll}
\hline Phenotype & $n$ & Week 1 & Week 2 \\
\hline Lean & 5 & Control diet $\longrightarrow$ \\
Obese & 6 & Control diet $\longrightarrow$ Fish oil diet $\longrightarrow$ Fish oil diet $\longrightarrow$ Fish oil diet + rosiglitazone \\
Lean & 5 & Control diet + rosiglitazone $\longrightarrow$ \\
Obese & 6 & Control diet + rosiglitazone $\longrightarrow$ Fish oil diet + rosiglitazone \\
Lean & 5 & Control diet $\longrightarrow$ \\
Obese & 6 & Control diet $\longrightarrow$ \\
Lean & 5 & Control diet + rosiglitazone $\longrightarrow$ \\
Obese & 6 & Control diet + rosiglitazone $\longrightarrow$
\end{tabular}

incubated with the same medium containing rosiglitazone or fenofibrate dissolved in DMSO (final concentration $0.2 \%$ ) at the concentrations indicated for $24 \mathrm{~h}$.

\section{Laboratory methods}

Plasma cholesterol and triglycerides were measured by enzymatic methods. HDL cholesterol was determined using polyanion precipitation. Plasma NEFA were quantitated by an enzymatic assay (Wako Chemicals, Neuss, Germany). Rat insulin was determined by RIA (Sensitive Rat Insulin RIA Kit; Linco Research, St Charles, MO, USA). RNA was isolated using the RNeasy Midi Kit (Qiagen, Hilden, Germany).

\section{Northern and slot blotting}

Total RNA $(10 \mu \mathrm{g})$ was separated on $1 \%$ agarose-formaldehyde gels. Northern blotting and hybridisation to fulllength rat Apoa4, Apoa5, Apoc3 and Acox1 cDNA probes $[1,33]$ or to a rat $28 \mathrm{~S}$ rRNA oligonucleotide $\left(5^{\prime}\right.$-AAT CCT GCT CAG TAC GAG AGG AAC CGC AGG 3') labelled with ${ }^{32} \mathrm{P}$ were carried out as described [25]. Apoa4, Apoa5, and Apoc3 mRNA abundance was determined by quantitative slot blotting [34] using 1, 2 and $4 \mu \mathrm{g}$ of RNA/sample and was standardised to $28 \mathrm{~S}$ rRNA.

The sequence of rat Apoa 5 was retrieved from the Rattus norvegicus chromosome 8 BAC CH230-416D7 (GenBank accession number AC135409). The promoter regions of human APOA5 [11] and rat Apoa5 were aligned using the AlignX program of the Vector NTI 9.1 Advance package (Invitrogen, Carlsbad, CA, USA).

\section{Western blotting}

Recently, ELISA methods have been developed for determination of apoAV levels in human plasma ([35-37] and F. G. Schaap et al., unpublished results); Western blotting [1] is the only technique currently available for measuring apoAV in rats. Cross-reactivity of antibodies employed in our own ELISA with rat apoAV was insufficient to allow quantification of apoAV in rat samples. Therefore, rat apoAV was assessed semiquantitatively by immunoblot analysis. Minced liver tissue was homogenised in PBS containing Protease Inhibitor cocktail (Roche, Mannheim, Germany) using a Potter-Elvehjem homogeniser. For apoAV detection, $5 \mu \mathrm{l}$ pooled serum or $250 \mu \mathrm{g}$ pooled total liver protein was separated on $1.5-\mathrm{mm}$ thick $10 \%$ polyacrylamide gels under denaturing and reducing conditions. Immunodetection of apoAV was performed as described previously [1]. Chemiluminescent signals were captured using a Lumilmager-F1 workstation (Roche) and quantified with LumiAnalyst software (Roche). Immunoblot analysis of apoAV in pooled plasma samples and liver homogenates was performed at least in triplicate. To confirm the results, samples from lean controls and all groups of obese rats were also analysed individually.

\section{Statistical analysis}

All results are presented as mean $\pm \mathrm{SD}$. The effects of obesity, rosiglitazone treatment and fish oil diet and their interactions were analysed by three-way ANOVA, followed by post hoc comparisons by Newman-Keul tests using the Statistica program package (Statsoft, Tulsa, OK, USA); $p$ values $<0.05$ were considered significant.

\section{Results}

Basal expression of Apoa5, Apoa4 and Apoc3

Plasma triglycerides were increased almost two-fold in obese Zucker rats when compared with lean littermates (Table 2, Fig. 2), but basal hepatic Apoa5 mRNA abundance was not altered, as determined by Northern 
Table 2 Effects of fish oil diet and rosiglitazone on weight gain, plasma lipids, insulin and NEFA in lean and obese Zucker rats

\begin{tabular}{|c|c|c|c|c|c|c|c|c|}
\hline \multirow[t]{3}{*}{ Rosiglitazone } & \multicolumn{4}{|c|}{ Obese Zucker rats (6 rats/group) } & \multicolumn{4}{|c|}{ Lean Zucker rats (5 rats/group) } \\
\hline & \multicolumn{2}{|c|}{ Without rosiglitazone } & \multicolumn{2}{|c|}{ With rosiglitazone } & \multicolumn{2}{|c|}{ Without rosiglitazone } & \multicolumn{2}{|c|}{ With rosiglitazone } \\
\hline & Control diet & Fish oil diet & Control diet & Fish oil diet & Control diet & Fish oil diet & Control diet & Fish oil diet \\
\hline Body weight (g) & $518 \pm 5^{\mathrm{c}}$ & $516 \pm 19^{c}$ & $552 \pm 11^{\mathrm{c}}$ & $542 \pm 47^{\mathrm{c}}$ & $370 \pm 28$ & $363 \pm 13$ & $372 \pm 24$ & $384 \pm 47$ \\
\hline Weight gain (g/day) & $4.8 \pm 0.6$ & $6.5 \pm$ & $7.4 \pm 0.8^{\mathrm{c}, \mathrm{f}}$ & $7.7 \pm 2^{\mathrm{b}, \mathrm{f}}$ & $3.9 \pm 3.2$ & $4.2 \pm 1.4$ & $4.2 \pm 1.1$ & $4.3 \pm 0.6$ \\
\hline Triglycerides (mmol/l) & $4.99 \pm 2.41^{\mathrm{a}}$ & $1.82 \pm 0.56^{\mathrm{f}}$ & $2.25 \pm 0.59^{\mathrm{f}}$ & $0.90 \pm 0.14^{\mathrm{f}}$ & $2.69 \pm 0.51$ & $0.42 \pm 0.07^{\mathrm{c}}$ & $1.12 \pm 0.41$ & $0.46 \pm 0.12^{\mathrm{c}}$ \\
\hline Cholesterol (mmol/l) & $5.19 \pm 0.98^{\mathrm{a}}$ & $4.08 \pm 0.8^{\mathrm{f}}$ & $4.44 \pm 1.09$ & $3.98 \pm 0.28^{\mathrm{f}}$ & $2.30 \pm 0.21$ & $1.73 \pm 0.13$ & $2.17 \pm 0.18$ & $2.02 \pm 0.39$ \\
\hline HDL cholesterol (mmol/l) & $1.76 \pm 0.88$ & $2.17 \pm 0.28^{\mathrm{c}}$ & $2.28 \pm 0.10^{\mathrm{c}}$ & $2.09 \pm 4^{\mathrm{c}}$ & $1.27 \pm 0.08$ & $1.06 \pm 0.05$ & $1.29 \pm 0.13$ & $1.24 \pm 0.13$ \\
\hline Insulin (mU/l) & $630 \pm 212^{\mathrm{a}}$ & $743 \pm 331^{\mathrm{a}}$ & $288 \pm 100^{\mathrm{d}}$ & $208 \pm 44^{\mathrm{d}}$ & $152 \pm 57$ & $98 \pm 15$ & $72 \pm 17$ & $75 \pm 16$ \\
\hline NEFA $(\mu \mathrm{mol} / \mathrm{l})$ & $814 \pm 202^{\mathrm{a}}$ & $521 \pm 122^{\mathrm{e}}$ & $372 \pm 141^{\mathrm{e}}$ & $207 \pm 18^{\mathrm{e}}$ & $389 \pm 95$ & $205 \pm 37^{\mathrm{c}}$ & $165 \pm 31^{\mathrm{b}}$ & $156 \pm 28^{\mathrm{b}}$ \\
\hline
\end{tabular}

Significant differences from lean control: ${ }^{\mathrm{a}} p<0.001 ;{ }^{\mathrm{b}} p<0.01 ;{ }^{\mathrm{c}} p_{\mathrm{f}}<0.05$

Significant differences from obese control: ${ }^{\mathrm{d}} p<0.001,{ }^{\mathrm{e}} p<0.01 ;{ }^{\mathrm{f}} p<0.05$

blotting (Fig. 1) and quantitative slot blotting (Fig. 2). Liver apoAV protein content was unaltered. Plasma apoAV levels appeared increased on Western blots of pooled samples (Figs. 1 and 2). Using samples from individual rats, an increase of $56 \%$ was observed but this was not statistically significant ( $p=0.083$, data not shown). As in earlier experiments [24, 25], basal hepatic Apoa4 mRNA abundance was significantly higher in obese Zucker rats compared with lean controls, while Apoc3 mRNA was unchanged (Figs. 1, 3).

Effects of rosiglitazone

Rosiglitazone significantly reduced plasma triglycerides in obese Zucker rats and induced an increase of about $70 \%$ in hepatic Apoa 5 mRNA in livers of lean and obese Zucker rats (Figs. 1 and 2, Table 2). Liver and plasma apoAV protein levels, however, tended to decrease, as judged by Western blot analysis of pooled samples
(Figs. 1 and 2). Similar changes were observed using samples of individual obese rats, but only the decline of plasma apoAV to $33 \pm 17 \%$ of control in obese rats receiving rosiglitazone + fish oil diet was significant ( $p=0.038$, data not shown). The ratio of plasma apoAV: Apoa 5 mRNA decreased significantly from $1.00 \pm 0.41$ in obese controls to $0.42 \pm 0.29(p=0.03)$ in obese rats treated with rosiglitazone. The ratio of liver apoAV:Apoa 5 mRNA decreased to $0.62 \pm 0.48$ (not significant). Rosiglitazone markedly improved insulin sensitivity in obese Zucker rats, as shown by major decreases in plasma insulin and NEFA (Table 2), but had no significant effects on plasma total cholesterol and HDL cholesterol in obese Zucker rats (Table 2).

To determine whether the increase in Apoa 5 mRNA was due to a direct effect of rosiglitazone on liver cells, primary rat hepatocytes were incubated with 2 or $4 \mu \mathrm{mol} / 1$ rosiglitazone (Fig. 4). Incubation with fenofibrate, which has been reported to enhance APOA5 transcription and mRNA levels several-fold in human hepatocytes $[10,11]$,
Fig. 1 Effects of rosiglitazone and fish oil diet on plasma and liver apoAV and on hepatic Apoa5, Apoa4 and Apoc3 mRNA in lean and obese Zucker rats. Pooled plasma and liver homogenate samples were analysed by Western blotting. Northern blots of pooled liver RNA were hybridised with rat Apoa5, Apoa4 and Apoc3 cDNA, and with a rat $28 \mathrm{~S}$ rRNA oligonucleotide (five or six animals per group). Co Control diet, $F O$ fish oil diet, $L$ lean Zucker rats, $O$ obese Zucker rats

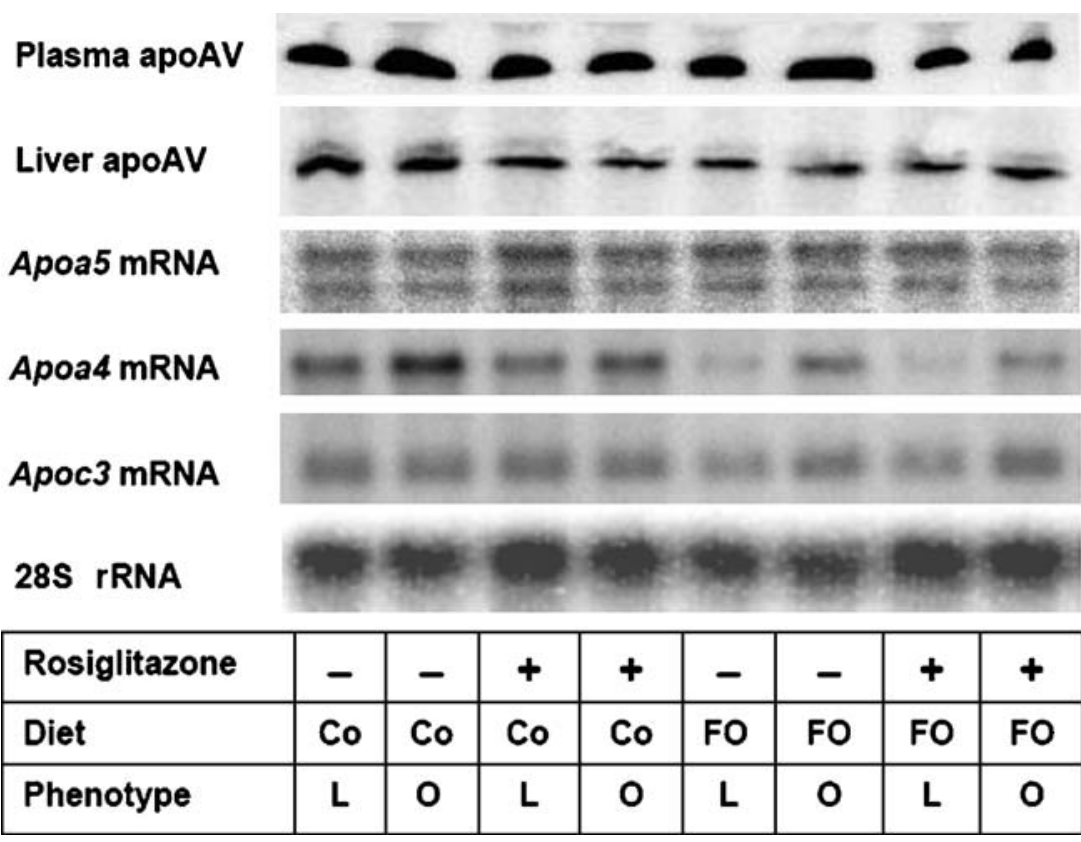


a

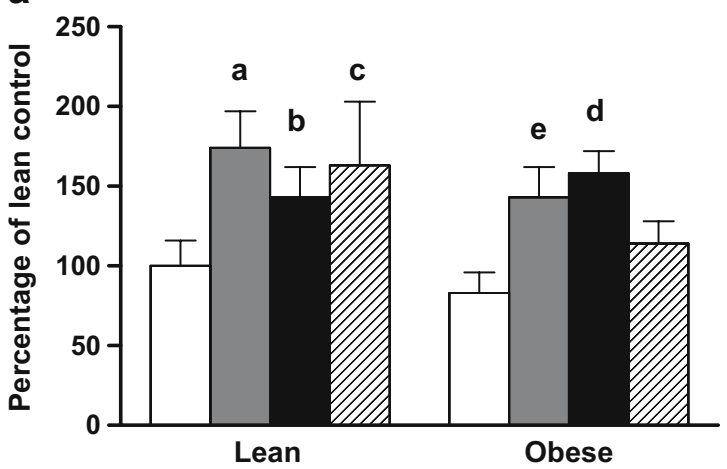

C

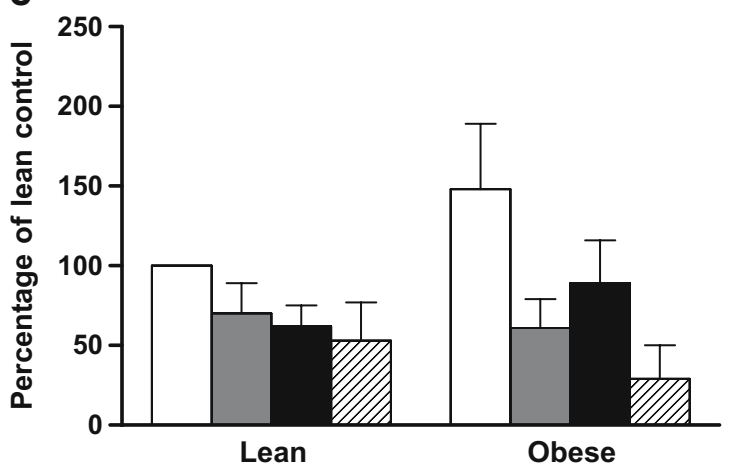

Fig. 2 Effect of rosiglitazone and fish oil diet on (a) hepatic Apoa5 mRNA, (b) liver apoAV protein, (c) plasma apoAV protein, and (d) plasma triglycerides in lean and obese Zucker rats. Lean or obese Zucker rats (five or six rats per group) received the control diet (white bars), control diet+rosiglitazone (grey bars), fish oil diet (black bars) or rosiglitazone+fish oil diet (hatched bars) as indicated in Table 1. Liver Apoa 5 mRNA was quantitated in triplicate in individual samples from five or six rats per group by slot blotting standardised to

was used as a control. Like human hepatocytes [10, 11], primary rat hepatocytes expressed little Apoa 5 mRNA compared with liver tissue. Rosiglitazone had no effect on Apoa 5 mRNA levels, suggesting that the upregulation of Apoa 5 mRNA observed in livers of rats receiving the drug occurred via indirect mechanisms. Unlike in human

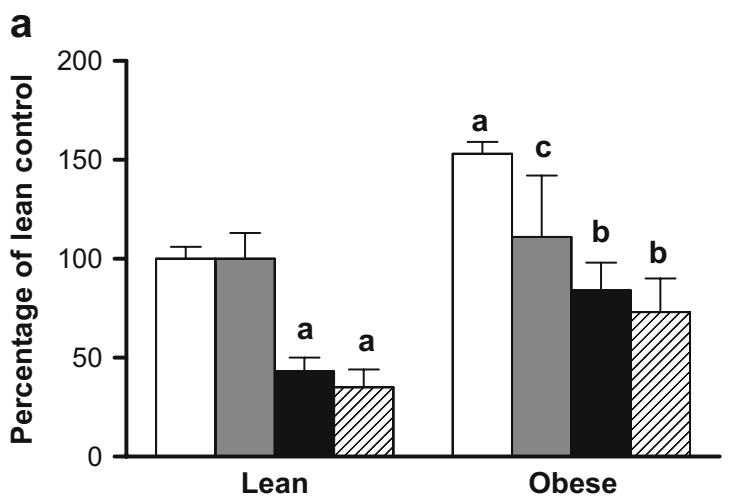

Fig. 3 Effects of rosiglitazone and fish oil diet on a hepatic Apoa4 and $\mathbf{b}$ Apoc 3 mRNA in lean and obese Zucker rats. Lean or obese Zucker rats (five or six per group) received the control diet (white bars), control diet+rosiglitazone (grey bars), fish oil diet (black bars) or rosiglitazone+fish oil diet (hatched bars) as indicated in Table 1. Liver Apoa4 and Apoc3 mRNA was quantitated in triplicate b
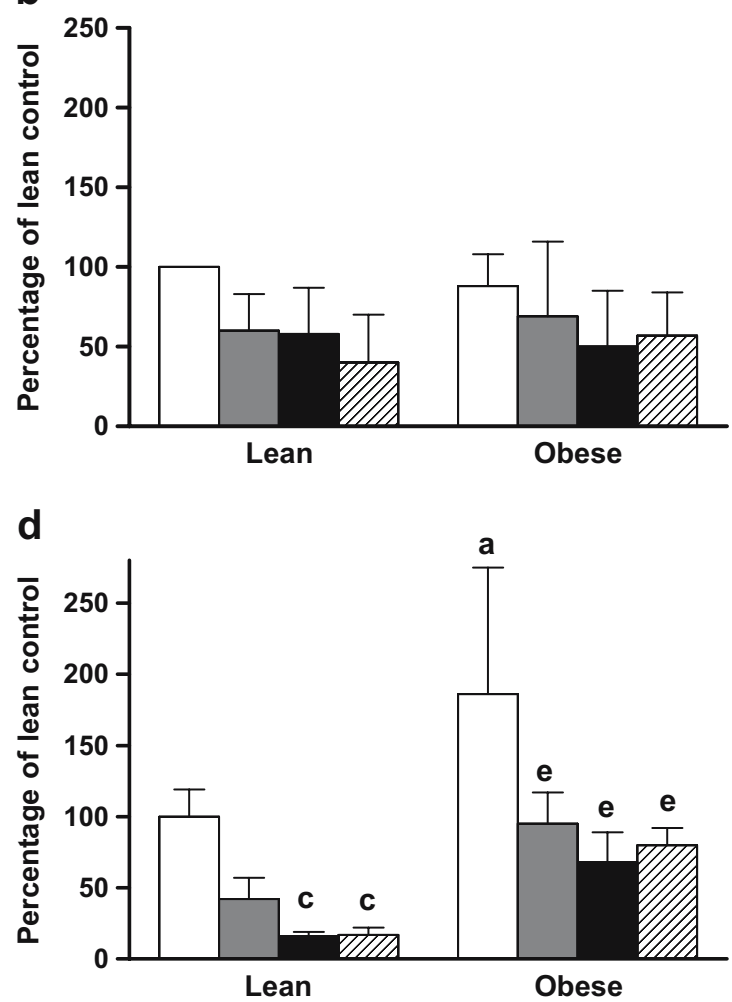

rat $28 \mathrm{~S}$ rRNA. Apoa $5 \mathrm{mRNA}$ and plasma triglyceride levels were analysed by three-way ANOVA and Newman-Keul tests and are given as mean \pm SD. Significant differences from lean control: ${ }^{\mathrm{a}} p<0.001 ;{ }^{\mathrm{b}} p<0.01 ;{ }^{\mathrm{c}} p<0.05$; significant differences from obese control: ${ }^{\mathrm{d}} p<0.01 ;{ }^{\mathrm{e}} p<0.05$. Liver and plasma apoAV protein were determined in pooled samples from each group by Western blotting (three or four independent analyses). Data are means \pm SD

hepatocytes, in rat hepatocytes fenofibrate failed to increase Apoa 5 mRNA, whereas induction of the PPAR $\alpha$ marker gene Acoxl was seen at a fenofibrate concentration of $100 \mu \mathrm{mol} / 1$, as described previously [38]. This result suggested that rat Apoa 5 may be insensitive to stimulation by PPAR agonists, and prompted us to examine the rat

\section{b}

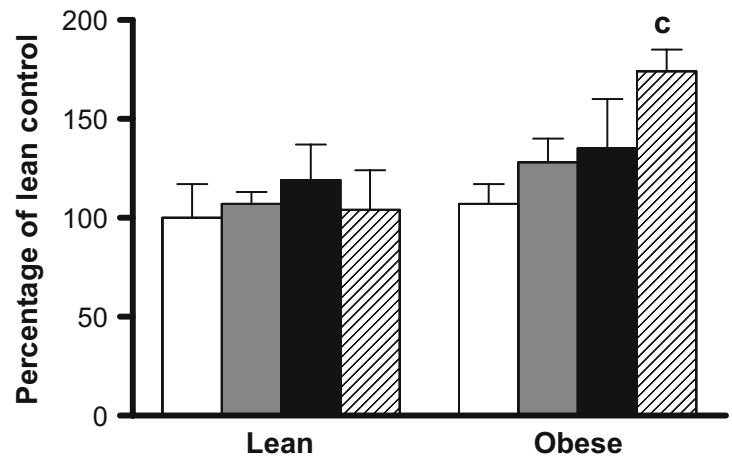

by slot blotting standardised to rat $28 \mathrm{~S}$ rRNA in individual samples from five or six rats per group. Data are means \pm SD. Significant differences from lean control: ${ }^{a} p<0.001$; significant differences from obese control: ${ }^{\mathrm{b}} p<0.001 ;{ }^{\mathrm{b}} p<0.01$ (three-way ANOVA, NewmanKeul tests) 
Fig. 4 Effects of rosiglitazone and fenofibrate on Apoa 5 mRNA in rat primary hepatocyte cultures. Hepatocyte cultures were incubated with rosiglitazone or fenofibrate for $24 \mathrm{~h}$. Total RNA was analysed by Northern blotting $(10 \mu \mathrm{g} /$ lane $)$ using rat Apoa 5 or Acox 1 cDNA and a $28 \mathrm{~S}$

rRNA. Rat liver RNA served as a control (first lane)
Apoa5 mRNA

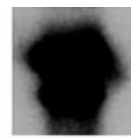

Acox 1 mRNA

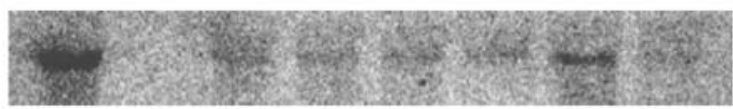

28S rRNA

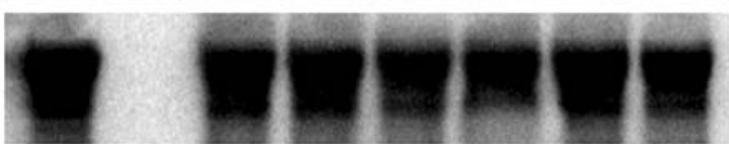

\begin{tabular}{|l|c|}
\hline Rosiglitazone $(\mu \mathrm{mol} / \mathrm{l})$ & - \\
\hline Fenofibrate $(\mu \mathrm{mol} / \mathrm{l})$ & - \\
\hline Sample & Liver \\
\hline
\end{tabular}

\begin{tabular}{|r|r|r|r|r|r|}
\hline 2 & 4 & - & - & - & - \\
\hline- & - & - & 20 & 100 & - \\
\hline \multicolumn{6}{|c|}{ Hepatocytes } \\
\hline
\end{tabular}

Apoa5 promoter for the presence of PPAR response elements (PPREs). Alignment of the rat Apoa5 promoter region with its human homologue revealed that the functional DR1 response element for PPAR contained in the human $A P O A 5$ promoter at position -257 to -271 harbours an insertion and is not fully conserved in the rat Apoa5 promoter (Fig. 5). No intact DR1 element was found within 1,000 nucleotides upstream of the transcription site of rat Apoa5. By contrast, the E-box element mediating the effect of insulin on the human $A P O A 5$ promoter [13] is conserved in the rat gene.

Regulation of hepatic Apoa4 expression by rosiglitazone differed clearly from that of Apoa5 (Figs. 1 and 3). Rosiglitazone normalised the increased basal Apoa4 mRNA levels in obese Zucker rats, but had no effect in lean rats. Hepatic Apoc3 mRNA levels were similar in lean and obese Zucker rats and were not affected by rosiglitazone administration in animals receiving the control diet.
Effects of fish oil diet

The fish oil diet decreased plasma triglycerides and significantly increased hepatic Apoa 5 mRNA in both lean and obese Zucker rats (Figs. 1 and 2, Table 2). Again, changes in liver and plasma apoAV protein concentrations did not follow changes in mRNA levels: plasma and liver apoAV in lean and obese Zucker rats (Figs. 1 and 2) and the ratios of plasma apoAV:Apoa5 mRNA and liver apoAV: Apoa 5 mRNA tended to decrease in obese rats (data not shown).

Apoa4 mRNA was significantly decreased, by about $50 \%$, in lean and obese Zucker rats receiving fish oil (Figs. 1 and 3). The fish oil diet did not alter Apoc3 mRNA significantly, as described previously (Figs. 1 and 3) [25]. Rosiglitazone treatment did not alter the response of hepatic Apoa4 and Apoa 5 mRNA to the fish oil diet. Obese
Fig. 5 Alignment of the promoter region of rat Apoa 5 and human APOA5. Relevant binding elements are boxed. Numbering refers to the position relative to the transcription start site

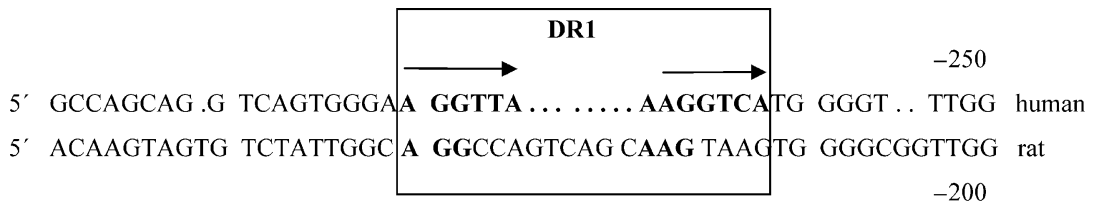

GAGAAACTGG GTGAGGAGTt CAGCCATCCC CC.GTAAAG. CTCCTGGGAA human TGgTCACAGG ATtGTGAGTT CTGCCCACTC TTAGTAAAgG CTCCAGGgAG rat $-150$ GCACTTCTCT ACTGGGGCAG CC.CCTGATA CCAGGGCA.C TCATTAACCC human CCACTTGTCT ACTGGGGCAG CCTCCTGAGG TTGGGGGAGC TTATTAACCC rat $-100$

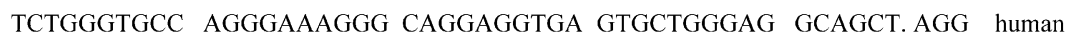
CGACAGCACC AGGAAAAGGG AAAAAGGCGA GTGCTAGTAG TCAGCTGAuG rat

E Box

$-50$

TCAACTTCTT TTGAACTTC ACGTGGTATT TACTCAGAGC AATTGGTGCC human

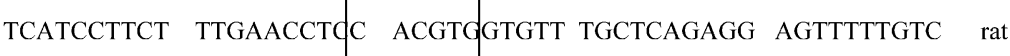

AGAGGCTCAG GGCCCTGGAG TATAAA GCAG AATGTCTGCT CTCTGTGCCC human AGACTCCCTG ACGCCTAGCG TATAAA ACAG AATGTCTGCT CTCTGTGCCC rat 
Zucker rats on rosiglitazone and the fish oil diet had higher Apoc3 mRNA levels than all other groups (Figs. 1 and 3).

\section{Discussion}

\section{Basal expression of Apoa5, Apoa4 and Apoc3}

Our study shows that the basal hepatic expression of Apoa 5 is not altered in hypertriglyceridaemic obese Zucker rats, whereas plasma apoAV may be moderately elevated. Obese Zucker rats develop hypertriglyceridaemia as a result of overproduction of triglyceride-rich VLDL enriched in apoE, apoC-III and apoA-IV by the liver [21,29]. ApoAV may be secreted in association with VLDL, thus accounting for the elevated plasma apoAV levels in obese Zucker rats overproducing VLDL. From a report that apoAV can lower the secretion rate of VLDL triglycerides [5], it appeared conceivable that reduced expression of Apoa5 in obese Zucker rats could contribute to the enhanced VLDL production. Our findings, however, suggest that apoAV is not involved in the pathogenesis of the secondary hypertriglyceridaemia associated with obesity and insulin resistance in these rats. In humans with hypertriglyceridaemia or familial combined hyperlipidaemia, polymorphisms in $A P O A 5$ show a strong association with plasma triglyceride levels across several ethnic groups $[2-4,39]$. ApoAV deficiency leads to severe hypertriglyceridaemia [40, 41]. Recent measurements of plasma apoAV in normolipaemic subjects revealed a weak negative correlation $[35,36]$ or a tendency towards a positive correlation of plasma apoAV and triglycerides (F. G. Schaap et al., unpublished results). On the other hand, in hypertriglyceridaemic subjects a significant positive correlation between plasma apoAV and triglyceride levels was observed (F. G. Schaap et al., unpublished results). Hypertriglyceridaemic patients with type 2 diabetes were reported to have fasting plasma apoAV levels similar to those of controls [37]. During postprandial hypertriglyceridaemia, plasma apoAV increased in these patients and remained unchanged in controls. These data and our findings in a naturally occurring animal model of hypertriglyceridaemia indicate that the relation of apoAV to plasma triglyceride levels is more complex than anticipated from the experiments in genetically modified mice.

Recently, transcriptional suppression of the human APOA5 and the mouse Apoa5 gene by insulin was reported, and insulin was shown to reduce plasma apoAV in euglycaemic clamp studies [13]. The E-box element mediating the effect of insulin on human APOA5 transcription via upstream stimulating factors is conserved in the rat gene. The liver of obese Zucker rats is severely insulin-resistant [42]. Thus, one might expect an increase in Apoa 5 mRNA and plasma apoAV in obese compared with lean Zucker rats. Our results of unaltered Apoa5 mRNA and liver protein in obese Zucker rats suggest that the effect of insulin on Apoa 5 mRNA may be blunted by other endocrine or metabolic changes in these animals.
In contrast to Apoa5, basal hepatic Apoa4 mRNA abundance was significantly higher in obese Zucker rats than in lean controls, as in earlier experiments [24, 25]. The mechanism of this increase, which is also seen in Wistar fatty rats and in rats made obese by a ventromedial lesion of the hypothalamus, is post-transcriptional and probably occurs at an early step of mRNA maturation [24, 25, 28]. The unchanged Apoc3 mRNA abundance in the liver of obese Zucker rats confirms the results of earlier studies [24, 25].

\section{Effects of rosiglitazone}

Administration of rosiglitazone increased hepatic Apoa 5 mRNA by about $70 \%$ but tended to decrease liver and plasma apoAV protein levels. Thus, neither liver nor plasma apoAV increased, whereas plasma triglyceride levels were lowered by more than $50 \%$. Therefore, apoAV does not appear to contribute to the marked decrease in plasma triglycerides induced by rosiglitazone in obese Zucker rats. In line with our observations are human data showing that pioglitazone significantly lowered plasma triglycerides in patients with type 2 diabetes, while plasma apoAV levels tended to decrease rather than to increase [43]. In transgenic mice overexpressing human $A P O A 5$, lipolysis of triglyceride-rich lipoproteins is enhanced and apoB and apoC-III are removed from plasma together with triglyceride-rich lipoprotein particles [6]. If apoAV is also cleared from the circulation during lipolysis, this may account for the discrepancy between plasma triglyceride levels and plasma apoAV during rosiglitazone treatment.

The upregulation of hepatic Apoa 5 mRNA by rosiglitazone is not mediated by a direct interaction of PPAR $\gamma$ with Apoa 5 because, in contrast to human $A P O A 5$, no intact PPRE was found in the rat gene. Thus, the regulation of APOA5 by $\operatorname{PPAR} \alpha$ appears to be species-specific, like the regulation by retinoic acid receptor $\alpha$-related orphan receptor (ROR- $\alpha$ ) [44]. Despite the apparent lack of a PPRE in the rat Apoa 5 promoter, fenofibrate effectively lowers triglycerides in Sprague-Dawley rats and in obese Zucker rats [22, 31]. This casts some doubt on the postulated importance of direct transactivation of APOA5 transcription by PPAR $\alpha$ for the hypolipidaemic effect of fenofibrate $[10,11]$.

The upregulation of Apoa5 mRNA by rosiglitazone appears to require signalling from other cells as it could not be reproduced in isolated hepatocytes. It cannot be explained by the improvement of insulin sensitivity or by anti-inflammatory effects of rosiglitazone, either of which should lead to a decrease in Apoa5 mRNA [13, 45]. Thiazolidinediones can alter lipid metabolism via molecules released from adipocytes, such as leptin, TNF- $\alpha$, resistin, adiponectin and NEFA [46]. Although there is no information on the regulation of apoAV by adipokines, our results show that Apoa5 is subject to regulation by fatty acids. Thus, the marked changes in plasma NEFA levels during rosiglitazone treatment may have contributed to the upregulation of ApoA5 mRNA.

Whereas rosiglitazone significantly induced hepatic Apoa 5 mRNA abundance, the apoAV protein levels in liver and 
plasma failed to increase. This suggests that, in addition to its well-documented transcriptional regulation, Apoa5 may be subject to regulation at a co- or post-translational level. Tight control of apoAV production at or after translation is also suggested by the contrast of the relatively high abundance of Apoa 5 mRNA in rat liver, resulting in hybridisation signals on Northern blots approaching the strength of Apoa4 signals in spite of very low hepatic apoAV protein levels. Translational regulation has been shown for Apoal, Apob and Apoe [47-49]. For apoB, co- and post-translational degradation is the main determinant of gene expression and has been studied extensively (reviewed in [49]).

In our study rosiglitazone reduced the increased Apoa4 mRNA abundance found in obese Zucker rats to a level indistinguishable from that in lean controls. Thus, the enhanced expression of Apoa4 in the liver of several rat models of obesity $[24,25]$ may be a consequence of insulin resistance. The absence of an effect of rosiglitazone on hepatic Apoc3 mRNA in lean and obese rats and on Apoa 4 mRNA in lean rats is confirmed by earlier studies $[22,28,31]$.

\section{Effects of fish oil diet}

The effects of the triglyceride-lowering fish oil diet on Apoa5 expression in Zucker rats resembled the effects induced by rosiglitazone: the abundance of hepatic Apoa 5 mRNA increased, whereas liver and plasma apoAV failed to increase. This argues against the involvement of apoAV in the lipid-lowering effect of $\omega$-3 PUFA. Similarly, liver apoAV failed to increase when Sprague-Dawley rats were fed a single meal per day of a fish oil diet for 4 days, while plasma triglycerides had declined more than two-fold already after the first meal (B. Hagerty and W. Strobl, unpublished results). The mechanisms underlying the hypolipidaemic actions of $\omega-3$ PUFA are incompletely understood at present. $\omega-3$ PUFA decrease the concentration and nuclear translocation of sterol regulatory element binding protein 1 (SREBP 1) (reviewed in [26]). As SREBP 1 represses APOA5 transcription [14], this may result in an increase in Apoa 5 mRNA. The apparently divergent regulation of liver Apoa 5 mRNA and protein by dietary $\omega-3$ PUFA provides a second example pointing to the possibility that co- or post-translational regulation of Apoa 5 may occur. $\omega-3$ PUFA are known to reduce hepatic apoB production by stimulating the post-endoplasmic reticulum presecretory pathway of apoB degradation [50].

In contrast to our earlier finding that the increased Apoa4 mRNA abundance in the liver of obese Zucker and Wistar rats was insensitive to transcriptional downregulation by dietary fish oil [24, 25], such a diet did reduce liver Apoa4 mRNA abundance in both lean and obese Zucker rats in the present study. In our earlier experiments, rat chow rather than a high-fat coconut oil diet served as a control, which may account for this difference.

In conclusion, this study shows that, at least in Zucker rats, neither the basal expression of Apoa 5 nor changes in Apoa 5 expression induced by a hypolipidaemic drug or a lipid-lowering diet are necessarily inversely related to plasma triglyceride levels. Moreover, Apoa 5 appears to be regulated not solely at the transcriptional level, but also during later stages of gene expression. This may be of importance for attempts to design lipid-lowering strategies exploiting the triglyceride-lowering effects of apoAV.

Acknowledgements The authors thank K. Herkner for lipid analysis, K. Blazey and S. Merich for tissue culture, and J. Graf for rat hepatocyte preparation. This study was supported by the Austrian Science Fund (grant 13649 BIO) and by the Austrian National Bank (grant 8830).

Duality of interest The authors are not aware of any duality of interest.

\section{References}

1. van der Vliet HN, Sammels MG, Leegwater AC et al (2001) Apolipoprotein A-V: a novel apolipoprotein associated with an early phase of liver regeneration. J Biol Chem 276:44512-44520

2. Pennacchio LA, Olivier M, Hubacek JA et al (2001) An apolipoprotein influencing triglycerides in humans and mice revealed by comparative sequencing. Science 294:169-173

3. Eichenbaum-Voline S, Olivier M, Jones EL et al (2004) Linkage and association between distinct variants of the APOA1 C3 A4 A5 gene cluster and familial combined hyperlipidemia. Arterioscler Thromb Vasc Biol 24:167-174

4. Talmud P, Hawe E, Martin S et al (2002) Relative contribution of variation within the APOC3 A4 A5 gene cluster in determining plasma triglycerides. Hum Mol Genet 11:3039-3046

5. Schaap FG, Rensen PC, Voshol PJ et al (2004) ApoAV reduces plasma triglycerides by inhibiting very low density lipoproteintriglyceride (VLDL-TG) production and stimulating lipoprotein lipase-mediated VLDL-TG hydrolysis. J Biol Chem 279: 27941-27947

6. Fruchart-Najib J, Bauge E (2004) Mechanism of triglyceride lowering in mice expressing human apolipoprotein A5. Biochem Biophys Res Commun 319:397-404

7. van der Vliet HN, Schaap FG, Levels JH et al (2002) Adenoviral overexpression of apolipoprotein $\mathrm{A}-\mathrm{V}$ reduces serum levels of triglycerides and cholesterol in mice. Biochem Biophys Res Commun 295:1156-1159

8. Merkel M, Loeffler B, Kluger M et al (2005) Apolipoprotein AV accelerates plasma hydrolysis of triglyceride rich lipoproteins by interaction with proteoglycan-bound lipoprotein lipase. J Biol Chem 280:21553-21560

9. van Dijk KW, Rensen PC, Voshol PJ, Havekes LM (2004) The role and mode of action of apolipoproteins CIII and AV: synergistic actors in triglyceride metabolism? Curr Opin Lipidol 15:239-246

10. Vu-Dac N, Gervois P, Jakel H et al (2003) Apolipoprotein A5, a crucial determinant of plasma triglyceride levels, is highly responsive to peroxisome proliferator-activated receptor alpha activators. J Biol Chem 278:17982-17985

11. Prieur X, Coste H, Rodriguez JC (2003) The human apolipoprotein $\mathrm{AV}$ gene is regulated by peroxisome proliferator-activated receptor-alpha and contains a novel farnesoid X-activated receptor response element. J Biol Chem 278:25468-25480

12. Schultze AE, Alborn WE, Newton RK, Konrad RJ (2005) Administration of a PPARalpha agonist increases serum apolipoprotein $\mathrm{A}-\mathrm{V}$ levels and the apolipoprotein A-V apolipoprotein C-III ratio. J Lipid Res 46:1591-1595

13. Nowak M, Helleboid-Chapman A, Jakel H et al (2005) Insulinmediated down-regulation of apolipoprotein A5 gene expression through the phosphatidylinositol 3-kinase pathway: role of upstream stimulatory factor. Mol Cell Biol 25:1537-1548 
14. Jakel H, Nowak M, Moitrot E et al (2004) The liver X receptor ligand T0901317 down-regulates APOA5 gene expression through activation of SREBP-1c. J Biol Chem 279:45462-45469

15. Steinmetz A, Barbaras R, Ghalim N, Clavey V, Fruchart JC, Ailhaud G (1990) Human apolipoprotein A-IV binds to apolipoprotein A-IA-II receptor sites and promotes cholesterol efflux from adipose cells. J Biol Chem 265:7859-7863

16. Steinmetz A, Utermann G (1985) Activation of lecithin:cholesterol acyltransferase by human apolipoprotein A-IV. J Biol Chem 260:2258-2264

17. Duverger N, Tremp G, Caillaud J et al (1996) Protection against atherogenesis in mice mediated by human apolipoprotein A-IV. Science 273:966-968

18. Verges BL, Vaillant G, Goux A, Lagrost L, Brun JM, Gambert P (1994) Apolipoprotein A-IV levels and phenotype distribution in NIDDM. Diabetes Care 17:810-817

19. Lingenhel A, Eder C, Zwiauer K et al (2004) Decrease of plasma apolipoprotein A-IV during weight reduction in obese adolescents on a low fat diet. Int J Obes Relat Metab Disord 28:1509-1513

20. Phillips MS, Liu Q, Hammond HA et al (1996) Leptin receptor missense mutation in the fatty Zucker rat. Nat Genet 13:18-19

21. Witztum JL, Schonfeld G (1979) Lipoproteins in the plasma and hepatic perfusates of the Zucker fatty rat. Diabetes 28:509-516

22. Chaput E, Saladin R, Silvestre M, Edgar AD (2000) Fenofibrate and rosiglitazone lower serum triglycerides with opposing effects on body weight. Biochem Biophys Res Commun 271:445-450

23. Mohan PF, Phillips FC, Cleary MP (1991) Metabolic effects of coconut, safflower, or menhaden oil feeding in lean and obese Zucker rats. Br J Nutr 66:285-299

24. Schuller E, Patel N, Item C et al (2000) The genetic background modifies the effects of the obesity mutation, 'fatty', on apolipoprotein gene regulation in rat liver. Int $\mathrm{J}$ Obes Relat Metab Disord 24:460-467

25. Strobl W, Knerer B, Gratzl R, Arbeiter K, Lin-Lee YC, Patsch W (1993) Altered regulation of apolipoprotein A-IV gene expression in the liver of the genetically obese Zucker rat. J Clin Invest 92:1766-1773

26. Clarke SD (2004) The multi-dimensional regulation of gene expression by fatty acids: polyunsaturated fats as nutrient sensors. Curr Opin Lipidol 15:13-18

27. Ribeiro A, Mangeney M, Cardot P et al (1991) Effect of dietary fish oil and corn oil on lipid metabolism and apolipoprotein gene expression by rat liver. Eur J Biochem 196:499-507

28. Inui Y, Keno Y, Fukuda K et al (1997) Modulation of apolipoprotein gene expression in fatty liver of obese rats: enhanced APOA-IV, but no APOB expression by a high sucrose diet. Int J Obes Relat Metab Disord 21:231-238

29. Pessah M, Salvat C, Wang SR, Infante R (1987) In vitro synthesis of apoA-IV and apoC by liver and intestinal mRNAs from lean and obese Zucker rats. Biochem Biophys Res Commun 142:78-85

30. Uchida E, Masumoto A, Sakamoto S, Koga S, Nawata H (1991) Effect of insulin, glucagon or dexamethasone on the production of apolipoprotein A-IV in cultured rat hepatocytes. Atherosclerosis 87:195-202

31. Lefebvre A, Peinado-Onsurbe J, Peinado-Onsurbe J et al (1997) Regulation of lipoprotein metabolism by thiazolidinediones occurs through a distinct but complementary mechanism relative to fibrates. Arterioscler Thromb Vasc Biol 17:1756-1764

32. Seglen PO (1976) Preparation of isolated rat liver cells. Methods Cell Biol 13:29-83

33. Radosavljevic M, Lin-Lee YC, Soyal SM et al (1992) Effect of sucrose diet on expression of apolipoprotein genes A-I, C-III and A-IV in rat liver. Atherosclerosis 95:147-156
34. Lin-Lee YC, Tanaka Y, Lin CT, Chan L (1981) Effects of an atherogenic diet on apolipoprotein $\mathrm{E}$ biosynthesis in the rat. Biochemistry 20:6474-6480

35. O'Brien PJ, Alborn WE, Sloan JH et al (2005) The novel apolipoprotein A5 is present in human serum, is associated with VLDL, HDL, and chylomicrons, and circulates at very low concentrations compared with other apolipoproteins. Clin Chem $51: 351-359$

36. Ishihara M, Kujiraoka T, Iwasaki T et al (2005) A sandwich enzyme-linked immunosorbent assay for human plasma apolipoprotein A-V concentration. J Lipid Res 46:2015-2022

37. Pruneta-Deloche V, Ponsin G, Groisne L, Fruchard-Najib F, Lagarde M, Moulin P (2005) Postprandial increase of apoAV concentrations in type 2 diabetic patients. Atherosclerosis 181:403-405

38. Lawrence JW, Li Y, Chen S et al (2001) Differential gene regulation in human versus rodent hepatocytes by peroxisome proliferator-activated receptor (PPAR) alpha. PPAR alpha fails to induce peroxisome proliferation-associated genes in human cells independently of the level of receptor expression. J Biol Chem 276:31521-31527

39. Endo K, Yanagi H, Araki J, Hirano C, Yamakawa-Kobayashi K, Tomura S (2002) Association found between the promoter region polymorphism in the apolipoprotein $\mathrm{A}-\mathrm{V}$ gene and the serum triglyceride level in Japanese schoolchildren. Hum Genet 111:570-572

40. Oliva CP, Pisciotta L, Li Volti G et al (2005) Inherited apolipoprotein A-V deficiency in severe hypertriglyceridemia. Arterioscler Thromb Vasc Biol 25:411-417

41. Marcais C, Verges B, Charriere S et al (2005) Apoa5 Q139X truncation predisposes to late-onset hyperchylomicronemia due to lipoprotein lipase impairment. J Clin Invest 115:2862-2869

42. Terrettaz J, Assimacopoulos-Jeannet F, Jeanrenaud B (1986) Severe hepatic and peripheral insulin resistance as evidenced by euglycemic clamps in genetically obese fa fa rats. Endocrinology 118:674-678

43. Nagashima K, Lopez C, Donovan D et al (2005) Effects of the PPAR gamma agonist pioglitazone on lipoprotein metabolism in patients with type 2 diabetes mellitus. J Clin Invest 115:1323-1332

44. Genoux A, Dehondt H, Helleboid-Chapman A et al (2005) Transcriptional regulation of apolipoprotein A5 gene expression by the nuclear receptor RORalpha. Arterioscler Thromb Vasc Biol 25:1186-1192

45. Khovidhunkit W, Duchateau PN, Medzihradszky KF et al (2004) Apolipoproteins A-IV and A-V are acute-phase proteins in mouse HDL. Atherosclerosis 176:37-44

46. Yu YH, Ginsberg HN (2005) Adipocyte signaling and lipid homeostasis: sequelae of insulin-resistant adipose tissue. Circ Res 96:1042-1052

47. Azrolan N, Odaka H, Breslow JL, Fisher EA (1995) Dietary fat elevates hepatic apoA-I production by increasing the fraction of apolipoprotein A-I mRNA in the translating pool. J Biol Chem 270:19833-19838

48. Srivastava RA, Srivastava N, Averna M et al (1997) Estrogen up-regulates apolipoprotein E (ApoE) gene expression by increasing ApoE mRNA in the translating pool via the estrogen receptor alpha-mediated pathway. J Biol Chem 272: 33360-33366

49. Fisher EA, Ginsberg HN (2002) Complexity in the secretory pathway: the assembly and secretion of apolipoprotein Bcontaining lipoproteins. J Biol Chem 277:17377-17380

50. Pan M, Cederbaum AI, Zhang YL, Ginsberg HN, Williams KJ, Fisher EA (2004) Lipid peroxidation and oxidant stress regulate hepatic apolipoprotein $\mathrm{B}$ degradation and VLDL production. J Clin Invest 113:1277-1287 